\title{
Bases para un modelo «pro-persistencia» estudiantil en educación superior aplicable en contextos socio-académicos desfavorables
}

\section{Bases for a "pro-persistence" student model in higher education applicable in unfavorable socio-academic contexts.}

DOI: $10.46932 / \mathrm{sfjdv} 2 \mathrm{n} 4-026$

Received in: March 1st, 2021

Accepted in: May 30th, 2021

\author{
Fernando Acevedo Calamet \\ Doctor CUM LAUDE (Universidad Internacional de La Rioja, España) \\ Prof. Adjunto. Responsable académico del Centro de Estudios sobre Políticas Educativas. \\ Centro Universitario Regional del Noreste, Universidad de la República, Uruguay. \\ Ituzaingó 667 (CP 40000), Rivera, Uruguay. \\ E-mail: face@cur.edu.uy
}

\section{RESUMEN}

La investigación que se reseña en este artículo se centró en dos focos problemáticos: (i) la afectación que las características estructurales del contexto socio-académico en el que se inscribe una organización de Educación Superior (ES), cuando su cualidad es desfavorable, produce en las condiciones inherentes al estudiante que ingresa, en especial en su disposición y motivación hacia el aprendizaje; (ii) la evaluación del grado en que esa afectación pone en riesgo la persistencia estudiantil. En el caso de Rivera, en el noreste de Uruguay, la estructura de oportunidades laborales y fundamentalmente educacionales terciarias - escasas y poco diversificadas- constituye un caldo de cultivo de eventos de riesgo de abandono de los estudios. El objetivo capital de la investigación fue aportar insumos teóricamente consistentes y empíricamente sustentados para la elaboración de un modelo «pro-persistencia» estudiantil en ES aplicable a contextos socio-académicos desfavorables y entonces superador, en su aplicabilidad, del modelo más aceptado en el actual mundo académico: el «Model of Institutional Action for Student Success» (MIASS) formulado por Tinto en 2012. En la investigación se tuvieron especialmente en cuenta algunos relevantes planteos teóricos y conceptuales sobre la temática, entre los que se destacan, por su profundidad y rigor, los más recientes de Tinto, Seidman, Kuh y Pascarella \& Terenzini. La investigación asumió un enfoque meso-estructural y una estrategia metodológica predominantemente cualitativa: análisis documental, entrevista en profundidad, grupo de discusión; también se aplicó una encuesta censal. El más relevante de los resultados alcanzados es que en lugares que, como Rivera, ofrecen pocas opciones de estudios superiores, las posibilidades de la persistencia estudiantil resultan notoriamente restringidas, ya que en esos casos la motivación intrínseca del estudiante hacia sus estudios suele ser débil: una considerable cantidad de estudiantes, al egresar de la Educación Media, decide cursar alguna de las pocas ofertas de ES existentes en su ciudad y no la que preferiría cursar si existiera esa opción. Esa débil motivación es, pues, el principal factor de riesgo de abandono, sobre todo en el primer año. Tal constatación es la base sustantiva sobre la que habrá de elaborarse un modelo «pro-persistencia» estudiantil alternativo al MIASS, en tanto aplicable en contextos socio-académicos desfavorables. Aquí radica la principal contribución que esta investigación puede ofrecer a organizaciones de ES inscriptas en contextos con oportunidades educacionales y laborales reducidas, tanto en cuanto a una inserción laboral atractiva (durante los estudios superiores o al finalizarlos) como, muy especialmente, a una oferta de ES escasa y poco diversificada.

Palabras clave: Persistencia estudiantil, Contextos desfavorables, Educación superior, Uruguay. 


\section{ABSTRACT}

The research reviewed in this article focused on two problem areas: (i) the affectation that the structural characteristics of the socio-academic context in which a Higher Education (HE) organization is inscribed, when its quality is unfavorable, produces in the inherent conditions of the entering student, especially in his/her disposition and motivation towards learning; (ii) the evaluation of the degree to which this affectation puts student persistence at risk. In the case of Rivera, in the northeast of Uruguay, the structure of labor and mainly tertiary educational opportunities -scarce and not very diversified- constitutes a breeding ground for dropout risk events. The main objective of the research was to provide theoretically consistent and empirically supported inputs for the development of a "pro-persistence" student model in HE applicable to unfavorable socio-academic contexts and thus surpassing, in its applicability, the most accepted model in the current academic world: the "Model of Institutional Action for Student Success" (MIASS) formulated by Tinto in 2012. The research especially took into account some relevant theoretical and conceptual approaches on the subject, among which the most recent ones by Tinto, Seidman, Kuh and Pascarella \& Terenzini stand out for their depth and rigor. The research assumed a mesostructural approach and a predominantly qualitative methodological strategy: documentary analysis, in-depth interview, discussion group; a census survey was also applied. The most relevant of the results obtained is that in places which, like Rivera, offer few options for higher education studies, the possibilities of student persistence are notoriously restricted, since in these cases the intrinsic motivation of students towards their studies is usually weak: a considerable number of students, upon graduating from high school, decide to study one of the few HE offers existing in their city and not the one they would prefer to study if that option existed. This weak motivation is, therefore, the main risk factor for dropping out, especially in the first year. This finding is the substantive basis on which an alternative "pro-persistence" student model to MIASS will have to be developed, as it is applicable in unfavorable socio-academic contexts. Herein lies the main contribution that this research can offer to HE organizations in contexts with reduced educational and employment opportunities, both in terms of an attractive labor market insertion (during or upon completion of higher education) and, especially, to a scarce and poorly diversified HE offer.

Key words: Student persistence, Unfavorable contexts, Higher education, Uruguay.

\section{INTRODUCCIÓN}

Investigar sobre la persistencia y sobre el abandono de los estudios en la Educación Superior (de aquí en adelante, ES) es fundamental para comprender cabalmente ambos fenómenos y en especial su impacto tanto en la vida social, académica y profesional de los estudiantes como en la dinámica de las propias organizaciones educativas (Souza do Prado, 2020).

En este contexto, en la actual producción académica orientada a analizar o a promover la persistencia estudiantil en ES existe un neto predominio de estudios que se enfocan en aquellos factores explicativos del abandono cuyo abordaje está al alcance de la acción directa de los centros educativos. El ejemplo paradigmático de este tipo de estudios es el que dio lugar al «Model of Institutional Action for Student Success» propuesto por Vincent Tinto (2012a), sin dudas el autor que, a la luz de la profusa cantidad de citas y alusiones que ha recibido, es desde hace varias décadas el referente teórico más reconocido en la temática. 
Sin embargo, la aplicabilidad de ese modelo y de otros muy afines a él -como el de Alan Seidman (2012), entre otros- es restringida. En efecto, este artículo presenta algunos resultados de una investigación de corte eminentemente cualitativo que ha constatado que en el caso de contextos socioacadémicos desfavorables -tal como los existentes en la región noreste de Uruguay, y en especial en la ciudad de Rivera- corresponde prestar especial atención a otros dos complejos de factores explicativos del abandono en ES cuyo estudio suele ser, en el mejor de los casos, marginal: los aspectos inherentes al estudiante al momento de su ingreso a la ES y las condiciones del contexto territorial en el que se inscribe el centro educativo - en los términos de Tinto (2012a), «internal commitments» y «external commitments» respectivamente-

\section{PROBLEMÁTICA PROPUESTA}

El acceso y la persistencia en la ES es motivo de creciente preocupación en el mundo occidental, en buena medida debido a la consolidación de nuevas relaciones entre las políticas educativas públicas y las condiciones emergentes del mercado laboral (Ferreira, 2020). Ahora bien, en los ámbitos geográficos que concentran la mayor parte de la actual producción académica sobre la problemática del abandono en ES las condiciones estructurales del contexto en el que se inscribe el centro educativo no parecen tener una influencia significativa en el truncamiento temprano de trayectorias académicas. Así lo evidencia una gran cantidad de obras recientes de gran aceptación en la comunidad científica occidental, entre las que se destacan las siguientes: en los EE.UU., Cabrera et al. (2014), Habley, Bloom y Robbins (2012), Kuh et al. (2010), Seidman (2012; 2004) y Tinto (2012a; 2012b; 1987); en Inglaterra, Merrill (2015); en Bélgica, Pinxten et al. (2015); en Australia, Krause et al. (2005) y McKenzie y Schweitzer (2001); en España, Figuera y Torrado (2014).

Seguramente ello responde a que en ciudades grandes y medianas de esos ámbitos geográficos la existencia de una muy amplia y diversificada oferta de estudios en ES posibilita una adecuada satisfacción de la demanda de los ingresantes a ese nivel, y entonces contribuye a la eficacia en la eventual implementación de planes, programas o acciones de retención escolar. Esta situación resulta muy favorable a la persistencia estudiantil, ya que la motivación intrínseca del estudiante hacia la opción académica elegida no se ve obturada o fragilizada al momento de comenzar sus estudios superiores. En estos casos los factores de riesgo de abandono de los estudios quedan restringidos a algunos aspectos inherentes al estudiante («internal commitments»: competencias académicas, atributos personales y actitudinales, entre otros) y a aquellos otros que pueden ser abordados desde la gestión de los propios centros educativos o de las instituciones que los rigen («institutional commitments»: calidad de la 
enseñanza, clima organizacional, curriculum, apoyo académico, social y económico, promoción de la integración social y del involucramiento académico, entre otros).

El modelo propuesto por Tinto (2012a) es muy claro al respecto. Tal como se ilustra en la Fig. 1 que se presenta a continuación, los elementos que aparecen encerrados en el círculo mayor-«institutional commitments»- constituyen el foco de sus análisis; de hecho, él mismo lo denominó «Modelo de Acción Institucional». A los elementos externos a ese círculo, en cambio, les dedica una atención tangencial, la cual se evidencia ya en el contenido del formulario de encuesta provisto por la National Survey of Student Engagement, publicado originalmente por Kuh et al. (2010), principal instrumento para la dotación de base fáctica al modelo.

Fig. 1. El modelo de acción institutional de Tinto: «Elements of a Model of Institutional Action»

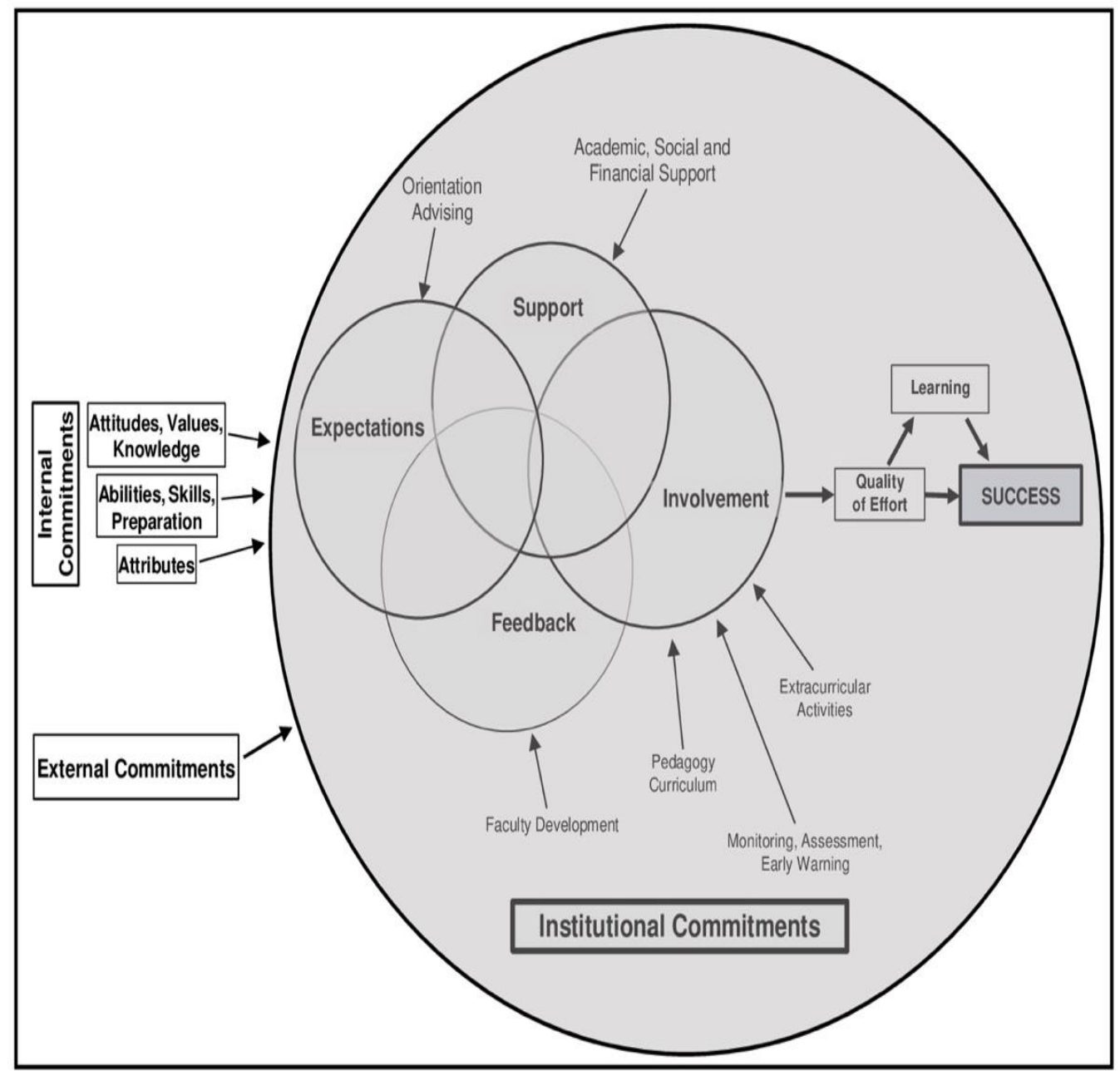

Fuente: elaboración propia a partir de Tinto (2012a)

A este respecto, la intuición que impulsó la ejecución de la investigación que aquí se reseña es que en el caso de centros de ES inscriptos en contextos socio-académicos desfavorables los elementos que en ese modelo aparecen incluidos en los rectángulos exteriores al círculo tienen una relevancia sustantiva en 
las trayectorias académicas de los estudiantes, sobre todo en su truncamiento temprano. Esos elementos pueden agruparse en dos conjuntos que, aunque diferentes, son interdependientes. Uno de ellos es el que comprende a las condiciones, situaciones y circunstancias inherentes al estudiante en el momento en el que decide su ingreso a un centro de ES -«internal commitments»-, tales como su preparación académica (en especial sus competencias cognitivas y conocimientos previos), sus atributos personales (el momento de su trayectoria vital, atributos de género y de clase, en especial sus capitales culturales y económicos), sus expectativas, actitudes y disposición hacia el aprendizaje. El otro conjunto comprende a las condiciones de corte eminentemente estructural propias del contexto territorial en el que se inscribe el centro -«external commitments»-: las estructuras de oportunidades educacionales (sobre todo la naturaleza, amplitud y diversificación de la oferta de estudios terciarios existente) y laborales (principalmente las posibilidades de inserción laboral durante los estudios superiores y/o una vez acreditada la ES).

En atención a esas consideraciones, la investigación se centró en dos focos problemáticos. Por una parte, en la afectación que las características estructurales del contexto en el que se inscriben las organizaciones de ES de Rivera - «external commitments»- produce en las condiciones inherentes al estudiante que ingresa a alguna de ellas -«internal commitments»-, en especial en sus actitudes, disposición y (des)motivación hacia el aprendizaje; por otra, en la evaluación de la medida en que esa afectación conduce a poner en riesgo la persistencia estudiantil. En ambos casos la convicción subyacente es que esos dos complejos fenoménicos («external commitments» e «internal commitments») presentan en Rivera configuraciones y contenidos muy diferentes que los existentes en lugares cuyos contextos socio-académicos son más favorables. Siendo así, la caracterización de aquella afectación resulta fundamental para la determinación y ponderación de los factores explicativos del abandono de los estudios en la ES en contextos socio-académicos desfavorables.

\section{CONTEXTO EN EL QUE SE INSCRIBE LA PROBLEMÁTICA PROPUESTA}

En el último decenio han crecido en forma exponencial los discursos y textos que destacan las crisis que anidan en la educación pública uruguaya. Además, desde fines de la década pasada la generalización del acceso a la ES y la reducción de la desafiliación en ese nivel constituyen una aspiración explícita de las autoridades del sector, en especial las de la Universidad de la República (Udelar), la institución de ES que conjuga la mayor tradición, volumen poblacional y relevancia social en el Uruguay contemporáneo. Tal aspiración se vuelve preocupación si se asume, por un lado, que "nuestro país se ubica entre los de la región con menor acceso a la educación superior, por debajo del promedio y lejos de otros con niveles similares de desarrollo" (Fernández y Cardozo, 2014, p. 123) y, por otro, que de cada tres 
estudiantes que ingresan a la ES casi dos abandonan sus estudios, la mitad de ellos entre el primer y el segundo año (Boado, Custodio y Ramírez, 2011). Otros estudios recientes (CIFRA, 2012) avalan esta afirmación para el caso de los centros educativos encargados de la formación de docentes para Educación Primaria y Educación Media, dependientes del Consejo de Formación en Educación (CFE) de la Administración Nacional de Educación Pública (ANEP).

La investigación que aquí se reseña ha constatado que la tasa de abandono en ES en Rivera es similar a la del promedio nacional. No obstante, esa similitud encubre condiciones, situaciones y circunstancias muy dispares, tanto si se consideran diferentes espacios geográficos como distintas organizaciones de ES: estructuras de oportunidades notoriamente disímiles, eventos de riesgo de índole e importancia relativa diferentes (Acevedo, 2013; 2011; 2009). Asimismo, por lo menos en el caso de la región noreste de Uruguay, la inadecuada estructura de oportunidades laborales y en especial educacionales terciarias -tanto en términos de escasez como de poca diversificación de la ofertaconstituye un caldo de cultivo de eventos de riesgo, sobre todo en el período de transición hacia la ES, tanto con respecto al acceso a ese nivel como al abandono de los estudios. Además, los efectos del abandono en ES son aún más preocupantes en el noreste de Uruguay, entre otras cosas porque es la región del país que presenta el mayor índice de pobreza y los indicadores más bajos de desarrollo de su población. En efecto, los cuatro departamentos que integran esta región (del total de 19 que conforman el territorio nacional) son los que presentan el menor Índice de Desarrollo Humano (Acevedo et al., 2013).

\section{OBJETIVOS}

Sobre la base de las consideraciones expuestas, la investigación tuvo como objetivo capital aportar insumos teóricamente consistentes y empíricamente sustentados para la elaboración de un modelo «propersistencia» estudiantil en primer año de ES aplicable a contextos socio-académicos desfavorables (como es el caso del existente en Rivera) y entonces superador, en términos de aplicabilidad, del modelo más aceptado en el actual mundo académico occidental: el «Model of Institutional Action for Student Success» formulado por Tinto (2012a). El principal de esos insumos -y, por ende, una de las contribuciones más destacables que ofrece la investigación que aquí se presenta- es la determinación de los principales factores intervinientes en el abandono de los estudios en el primer año de ES en los tres centros educativos públicos de ese nivel existentes en Rivera: el Centro Universitario de Rivera (Udelar), el Centro Regional de Profesores del Norte y el Instituto de Formación Docente de Rivera (estos últimos dependientes del CFE de la ANEP).

Los objetivos específicos establecidos para el cabal cumplimiento de ese objetivo general son los que a continuación se describen: 
(a) Determinar la actual magnitud del fenómeno de abandono de los estudios en el primer año de la ES en los tres centros públicos de ese nivel existentes en Rivera.

(b) Identificar y analizar las principales características de las condiciones y circunstancias que en la actualidad más inciden -y el modo diferencial en que lo hacen- en la decisión de los estudiantes de abandonar sus estudios en el primer año de la ES en cada uno de esos tres centros educativos.

(c) Determinar las condiciones contextuales (externas) de mayor incidencia en la decisión de los jóvenes de iniciar estudios superiores en Rivera, con énfasis en el análisis de las particularidades demóticas, socioculturales y económicas de la región noreste de Uruguay y de la estructura de oportunidades existente.

(d) Determinar las condiciones y características de los ingresantes a la ES (auto-percepción de su preparación académica y sus competencias -conocimientos específicos, habilidades, actitudes-, atributos -de género, de clase social, estructura de capitales-, preferencias, expectativas, intereses económicos, de prestigio, de socialidad-) que más inciden en su decisión de iniciar estudios en Rivera, en especial los asociados a la dimensión motivacional intrínseca.

\section{FUNDAMENTOS TEÓRICOS CONSIDERADOS}

En la formulación y en el diseño de la investigación se tuvieron especialmente en cuenta algunos relevantes planteos teóricos y conceptuales sobre la temática indagada, que por razones de espacio aquí no detallaremos. Entre ellos se destacan, por su profundidad y rigor, los incluidos en algunas obras publicadas en los EE.UU. en el último decenio (Choy, 2001; Kuh et al., 2005; Pascarella y Terenzini, 2005; Rumberger, 2004; Seidman, 2012; Shavit, Arum y Gamoran, 2007; Tinto, 2013) y otras en España (Casanova, 2007; Figuera y Torrado, 2014; Zabalza, 2002). Asimismo, se prestó especial atención a la producción nacional existente al respecto, tanto por su consistencia teórico-conceptual como por su particular anclaje empírico. En este sentido, se destacan las siguientes obras, casi todas producidas por investigadores de la Facultad de Ciencias Sociales de la Udelar: Acevedo (2014; 2011; 2009), Boado, Custodio y Ramírez (2011), Boado y Fernández (2010), Cardozo (2013), Cardozo et al. (2014), Fernández (2010), Fernández (coord. y ed., 2010), Fernández y Cardozo (2013), Mancebo (2010; 2000).

\section{METODOLOGÍA}

La investigación asumió un enfoque meso-estructural, esto es, un abordaje explicativo que sobre la base de la priorización de la consideración de los aspectos locales y organizacionales (por encima de los microsociales y los macrosociales, aunque sin desconocerlos) conjuga una "focalización en el papel de las organizaciones escolares sobre el comportamiento (en este caso la desafiliación) [con] los enfoques 
que destacan la relación entre las características de la localidad en términos de población [...] o de marginación socio-económica" (Fernández, 2010, p. 29).

Para la producción de información necesaria para dar cuenta del problema de investigación formulado se dispuso una estrategia predominantemente cualitativa, con apelación a variadas técnicas de producción de información: análisis documental, entrevista en profundidad, grupo de discusión. También se aplicó la técnica de encuesta (censal). A continuación se describen someramente las técnicas de producción de información empleadas y su contexto de aplicación.

La técnica de análisis documental se aplicó con vistas al cumplimiento del primero de los objetivos específicos propuestos: determinar la actual magnitud del fenómeno de abandono de los estudios en el primer año de la ES en los tres centros públicos de ese nivel existentes en Rivera. Comprendió el relevamiento, registro, procesamiento y análisis de documentos administrativos de cada uno de los tres centros educativos considerados.

La técnica de entrevista en profundidad se aplicó a 70 informantes calificados que fueron previamente categorizados según los cinco «tipos» que a continuación se describen:

i. 6 personas con conocimiento profundo y/o capacidad de influencia en los procesos de toma de decisiones de cada sector productivo local en el último decenio. La aplicación de estas entrevistas estuvo orientada al cumplimiento del objetivo específico (c) descrito antes.

ii. 7 actores con conocimiento profundo de la situación de la ES en la región y de los cambios acaecidos en el sector en el último decenio. La aplicación de estas entrevistas estuvo orientada al cumplimiento del objetivo específico (b) descrito antes.

iii. 9 actores con conocimiento profundo de alguno(s) de los tres centros de ES estudiados. La aplicación de estas entrevistas también se orientó al cumplimiento del objetivo específico (b).

iv. 24 jóvenes que, habiendo comenzado sus estudios en alguno de esos tres centros en 2014, continuaban estudiando allí una vez iniciado el tercer año lectivo (en 2016). La aplicación de estas entrevistas estuvo orientada al cumplimiento de los objetivos específicos (b) y (d).

v. 24 jóvenes de la misma cohorte que abandonaron sus estudios antes de transcurridos tres meses del inicio del tercer año lectivo de ES. Al igual que en el caso precedente, la aplicación de estas entrevistas también se orientó al cumplimiento de los objetivos específicos (b) y (d) ${ }^{1}$.

La técnica de encuesta censal se aplicó a los informantes de los «tipos» (iv) y (v), y estuvo orientada al cumplimiento del objetivo específico (d).

\footnotetext{
${ }^{1}$ En los dos «Apéndices» que se incluyen al final del presente texto se presentan las tablas de validación técnica de las pautas
} de las entrevistas en profundidad aplicadas a los informantes de los «tipos» (iv) y (v). 
La técnica de grupo de discusión se aplicó a un grupo integrado por seis de los doce informantes del «tipo» (iv) entrevistados (dos estudiantes de cada uno de los tres centros de ES de Rivera que, habiendo comenzado sus estudios en uno de esos centros en el año 2014, continuaban allí a julio de 2016) y seis de los doce informantes del «tipo» (v) entrevistados (dos estudiantes de cada uno de esos centros que, habiendo comenzado sus estudios en 2014, a julio de 2016 los habían abandonado). La selección de esos doce informantes se realizó en función del análisis de la información aportada en situación de entrevista en profundidad.

\section{RESULTADOS}

El más relevante de los resultados alcanzados en la investigación es que en aquellos lugares en los que se ofrecen pocas opciones de estudios en ES, como es el caso de Rivera, las posibilidades de la persistencia estudiantil resultan notoriamente restringidas. Ello se debe a que en lugares con esas características la motivación intrínseca del estudiante hacia el cursado de estudios superiores en alguna de esas escasas opciones disponibles es débil. En definitiva, una considerable cantidad de estudiantes, al egresar de la Educación Media Superior (o Bachillerato), toma la decisión de cursar alguna de las pocas «carreras» de ES que se ofrecen en el ámbito local, y no la que preferirían cursar si existiera esa opción en la oferta existente. En consecuencia, la débil motivación intrínseca de la gran mayoría de los estudiantes que inician estudios de ES en ese tipo de lugares se erige como el más importante factor de riesgo de abandono de los estudios, sobre todo en el primer año.

Por otra parte, de las entrevistas en profundidad efectuadas a los informantes calificados de los «tipos» (iv) - estudiantes persistentes-y (v) - estudiantes no persistentes- emergió con nitidez la existencia de una condición de carácter cultural: en su amplia mayoría esas personas no han tomado en consideración la posibilidad de cursar sus estudios superiores en ciudades en las que la oferta existente es más amplia y diversificada, como por ejemplo Montevideo (capital de Uruguay) o Porto Alegre (capital del Estado de Rio Grande do Sul, Brasil), ambas situadas a 500 quilómetros de distancia de Rivera; aquellas personas que en algún momento consideraron esa posibilidad de todos modos decidieron quedarse en su lugar de residencia, en algunos casos aduciendo razones económicas y en otros cuestiones afectivas. En cualquiera de los casos, predomina en ellos una suerte de mentalidad reticente a alejarse de su lugar, así como desconocimiento de la existencia de becas de apoyo económico orientadas a posibilitar ese traslado, residencias estudiantiles, etcétera.

Por último, a diferencia de la situación existente en lugares cuyos contextos socio-académicos son favorables -sobre todo por presentar una oferta de ES suficientemente amplia y diversificada-, en lugares como Rivera es muy difícil y poco viable que los factores de riesgo de abandono de los estudios 
mencionados antes puedan ser enfrentados por medio de acciones promovidas por la gestión académica y organizacional de los propios centros educativos. Ese tipo de acciones es, precisamente, el que aparece ocupando un rol protagónico en los dos modelos de retención escolar (o «pro-persistencia») actualmente más aceptados en el campo académico internacional: los ya aludidos «Model of Institutional Action for Student Success» (Tinto, 2012a) y el muy afín «Retention Formula and Model for Student Success» (Seidman, 2012).

\section{CONCLUSIONES Y CONTRIBUCIONES PARA EL TEMA}

En concordancia con lo destacado precedentemente, los modelos precitados no son del todo válidos ni aplicables para el caso de centros de ES que, como es el caso de los afincados en Rivera, están inscriptos en contextos institucionales de escasa y poco diversificada oferta de estudios de ES; en rigor, sólo resultarían idóneos y eficaces para orientar la promoción de la persistencia (o la retención) de aquellos pocos estudiantes cuyas preferencias de estudios en ES coinciden con alguno de los que se ofrecen localmente, es decir, aquellos que poseen motivación intrínseca orientada hacia la opción de estudios superiores elegida.

En conclusión, los resultados preliminares alcanzados hasta este momento constituyen las bases sustantivas sobre las que habrá de elaborarse un modelo alternativo «pro-persistencia» estudiantil aplicable en contextos socio-académicos desfavorables, en el que se privilegiará la consideración de los aspectos que en el «Model of Institutional Action for Student Success» (Tinto, 2012a) aparecen ubicados fuera del gran círculo central: «internal commitments» y «external commitments».

Aquí radica, pues, la principal contribución que la investigación esbozada puede ofrecer a aquellas organizaciones de ES inscriptas en contextos caracterizados por estructuras de oportunidades educacionales y laborales de escasa amplitud, tanto en lo atinente a las reducidas posibilidades de aspirar a una inserción laboral atractiva (durante los estudios superiores o bien al finalizarlos) como, muy especialmente, a una oferta de estudios superiores escasa y poco diversificada. 


\section{REFERENCIAS BIBLIOGRÁFICAS}

Acevedo, F. (2009). El Centro Universitario de Rivera, impulsor del sistema nervioso de la región. Análisis, diagnosis, prognosis. Montevideo: Universidad de la República.

Acevedo, F. (2011). Dicen. Calidad educativa y gobernabilidad en un instituto de formación docente. Montevideo: erga e omnes ediciones.

Acevedo, F. (2013). Abandono y riesgos de abandono en la Educación Superior en el interior del país. Un análisis provisional. Ponencia presentada en el Seminario-taller regional Transiciones entre ciclos, riesgos y desafiliación en la Educación Media y Superior de Uruguay, Rivera: Centro Universitario de Rivera, Universidad de la República, 2 de octubre.

Acevedo, F. (2014). Una aproximación a la desafiliación educativa en la transición hacia la Educación Superior en el interior de Uruguay. Páginas de Educación, 7(1), 131-148.

Acevedo, F., Fernández, T., Domínguez, M. E., Mancebo, M. E., Menni, G., Nossar, K., Ocaño, J., Patrón, R., Porta, M., Ríos, Á. y Viera, P. (2013). Centro de Estudios sobre Politicas Educativas. Proyecto para su constitución en el Centro Universitario de la Región Noreste de la Universidad de la República. Montevideo: Universidad de la República.

Boado, M., Custodio, L. y Ramírez, R. (2011). La deserción estudiantil universitaria en la UDELAR y en Uruguay entre 1997 y 2006. Montevideo: Comisión Sectorial de Investigación Científica, Universidad de la República.

Boado, M. y Fernández, T. (2010). Trayectorias académicas y laborales de los jóvenes en Uruguay. El panel PISA 2003-2007. Montevideo: Facultad de Ciencias Sociales, Universidad de la República.

CIFRA (2012). Estudio de los factores que influyen en la duración de las carreras de formación docente. Montevideo: Consejo de Formación en Educación, Administración Nacional de Educación Pública.

Cabrera, A., Pérez, P. y López, L. (2014). Evolución de perspectivas en el estudio de la retención universitaria en los EE.UU.: bases conceptuales y puntos de inflexión. En Figuera, F. (Ed.), Persistir con éxito en la universidad: de la investigación a la acción (pp. 15-40). Barcelona: Laertes.

Cardozo, S. (2013). Transición y desafiliación. Ponencia en el Seminario Transiciones entre ciclos, riesgos y desafiliación en la Educación Media y Superior de Uruguay. Montevideo: Facultad de Ciencias Sociales, Universidad de la República, 15 de julio.

Cardozo, S., Fernández, T., Míguez, M. y Patrón, R. (2014). Transición entre ciclos. Marco analítico. En Fernández, T. y Ríos, Á. (Eds.), Transiciones, riesgos de desafiliación y políticas de inclusión en la Educación Media y Superior de Uruguay (pp. 21-40). Montevideo: Comisión Sectorial de Investigación Científica, Universidad de la República.

Casanova, M. A. (2007). Evaluación y calidad de centros educativos. Madrid: La Muralla.

Choy, S. (2001). Students whose parents did not go to college. Postsecondary access, persistence and attainment. Washington, D. C.: National Center for Education Statistics, U. S. Department of Education. 
Fernández, T. (2010). Enfoques para explicar la desafiliación. En T. Fernández (coord. y ed.), La desafiliación en la Educación Media y Superior de Uruguay: conceptos, estudios, políticas (pp. 27-40). Montevideo: Comisión Sectorial de Investigación Científica, Universidad de la República.

Fernández, T. (coord. y ed.) (2010). La desafiliación en la Educación Media y Superior de Uruguay: conceptos, estudios, políticas. Montevideo: Comisión Sectorial de Investigación Científica, Universidad de la República.

Fernández, T. y Cardozo, S. (2013). Acceso y persistencia en el tránsito a la Educación Superior en los estudiantes uruguayos evaluados por PISA en 2003. Inédito.

Fernández, T. y Cardozo, S. (2014). Acceso y persistencia en el tránsito a la educación superior en la cohorte de estudiantes uruguayos evaluados por PISA en 2003. En Fernández, T. y Ríos, A. (eds.), El tránsito entre ciclos en la Educación Media y Superior de Uruguay (pp. 123-146). Montevideo: Comisión Sectorial de Investigacion Científica, Universidad de la República.

Ferreira, I. (2020). Economic changes and investment in higher education in Brazil 2000-2015. South Florida Journal of Development, 1(3), 89-105. DOI: 10.46932/sfjdv1n3-004

Figuera, P. y Torrado, M. (2014). Análisis longitudinal del proceso de transición a la universidad. Estudio de un caso. En Figuera, F. (Ed.), Persistir con éxito en la universidad: de la investigación a la acción (pp. 97-112). Barcelona: Laertes.

Habley, W., Bloom, J. y Robbins, S. (2012). Increasing Persistence. Research-Based Strategies for College Student Success. San Francisco: Jossey-Bass.

Krause, K.-L., Hartley, R., James, R. y McInnis, C. (2005). The First Year Experience in Australian Universities: Findings from a Decade of National Studies. Melbourne: Department of Education, Science and Training, Australian Government - Centre for the Study of Higher Education, University of Melbourne.

Kuh, G., Kinzie, J., Schuh, J., Whitt, E. \& Associates (2010). Student Success in College. Creating Conditions that Matter. San Francisco: Jossey-Bass.

Mancebo, M. E. (2000). El Sistema Educativo Uruguayo: Estudio, Diagnóstico y Propuestas Públicas para el sector. Montevideo: Banco Interamericano de Desarrollo.

Mancebo, M. E. (2010). Políticas de inclusión educativa en el Cono Sur: Argentina, Chile y Uruguay 2005-2009. Toronto: Latin American Studies Association Congress.

McKenzie, K. y Schweitzer, R. (2001). Who succeeds at university? Factors predicting academic performance in first year Australian university students. Higher Education Research \& Development, 20(1), 21-33.

Merrill, B. (2015). Determined to stay or determined to leave? A tale of learner identities, biographies and adult students in higher education. Studies in Higher Education, 40(1), 1859-1871.

Pascarella, E. y Terenzini, P. (2005). How College Affects Students. Vol. 2: A Third Decade of Research. San Francisco: John Willey \& Son. 
Pinxten, M., De Fraine, B., Van Den Noortgate, W., Van Damme, J., Boonen, T. y Vanlaar, G. (2015). 'I choose so I am': a logistic analysis of major selection in university and successful completion of the first year. Studies in Higher Education, 40(1), 1919-1946.

Rumberger, R. (2004). Why Students Drop Out of School? En Orfield, G., Dropouts in America. Confronting the Graduation Rate Crisis. Cambridge: Harvard Education Press.

Seidman, A. (2004). Retention Slide Show. www.cscsr.org/docs/RetentionFormula2004a_files/frame.htm.

Seidman, A. (2012). Taking Action. A Retention Formula and Model for Student Success. En Seidman, A. (ed.), College Student Retention. Formula for Student Success (pp. 267-284). Plymouth: Rowman \& Littlefield.

Shavit, Y., Arum, R. y Gamoran, A. (2007). Stratification in Higher Education. Stanford: Stanford University Press.

Souza do Prado, A. P. (2020). Panorama of the evasion in the graduation courses of DCH - Campus IX, period 2012 to 2016. South Florida Journal of Development, 1(3), 176-184. DOI: 10.46932/sfjdv1n3-011

Tinto, V. (1987). El abandono de los estudios superiores. Una nueva perspectiva de las causas del abandono y su tratamiento. México, D. F.: UNAM-ANUIES.

Tinto, V. (2012a). Moving from Theory to Action. A Model of Institutional Action for Student Success. En Seidman, A. (ed.), College Student Retention. Formula for Student Success, pp. 251-266. Plymouth: Rowman \& Littlefield.

Tinto, V. (2012b). Completing College: rethinking institutional action. Chicago: The University of Chicago Press.

Zabalza, M. (2002). La enseñanza universitaria: el escenario y sus protagonistas. Madrid: Narcea. 


\section{APÉNDICE 1}

(A) Tabla de validación técnica de la pauta de la entrevista en profundidad aplicada a los informantes del «tipo» (iv)

\begin{tabular}{|c|c|c|c|c|c|}
\hline \multicolumn{2}{|c|}{$\begin{array}{l}\text { OBJETIVO DE } \\
\text { INVESTIGA- } \\
\text { CIÓN }\end{array}$} & $\begin{array}{l}\text { TÉCNICA } \\
\text { APLICAD } \\
\quad \text { A }\end{array}$ & $\begin{array}{l}\text { INSTRU- } \\
\text { MENTO } \\
\text { APLICADO }\end{array}$ & $\begin{array}{l}\text { VARIABLES } \\
\text { CONSIDE- } \\
\text { RADAS }\end{array}$ & INDICADORES (o PREGUNTAS) \\
\hline \multirow[t]{14}{*}{$\stackrel{(b}{(b}$} & \multirow{14}{*}{$\begin{array}{c}\text { Identificar } \\
\text { y analizar } \\
\text { las } \\
\text { principales } \\
\text { característi } \\
\text {-cas de las } \\
\text { condiciones } \\
, \\
\text { situaciones } \\
\text { y } \\
\text { circunstan- } \\
\text { cias que } \\
\text { más inciden } \\
\text {-y el modo } \\
\text { diferencial } \\
\text { en que lo } \\
\text { hacen- en } \\
\text { la decisión } \\
\text { de } \\
\text { abandono } \\
\text { voluntario } \\
\text { de los } \\
\text { estudios en } \\
\text { el 1 }{ }^{\text {er }} \text { año de } \\
\text { la ES en } \\
\text { cada uno de } \\
\text { los tres } \\
\text { centros } \\
\text { educativos } \\
\text { considerad } \\
\text { os }\end{array}$} & \multirow{14}{*}{\begin{tabular}{|c} 
Entrevista \\
en \\
profundidad \\
a muestra de \\
24 \\
informantes \\
del tipo (iv): \\
8 jóvenes de \\
cada uno de \\
los 3 centros \\
considerado \\
s que \\
iniciaron allí \\
sus estu- \\
dios en 2014 \\
y \\
continuaron \\
allí una vez \\
iniciado el \\
$3^{\text {er año }}$ \\
lectivo. \\
[La \\
aplicación de \\
estas entre- \\
vistas es pos- \\
terior a la en- \\
cuesta censal \\
a estos \\
informan-tes, \\
y en la que se \\
aplicó el \\
módulo del \\
formulario - \\
de la NSSE]
\end{tabular}} & \multirow{10}{*}{\begin{tabular}{|} 
Pauta de \\
semi- \\
estructuració \\
$n$ de \\
entrevista en \\
profundidad
\end{tabular}} & \multirow{2}{*}{$\begin{array}{c}\text { Trayectoria } \\
\text { y } \\
\text { preparación } \\
\text { pre-terciaria }\end{array}$} & $\begin{array}{l}\text { ¿Dónde cursaste } 6^{\circ} \text { año de EMS? ¿En qué orientación? } \\
\text { ¿Recuerdas por qué elegiste esa orientación? }\end{array}$ \\
\hline & & & & & $\begin{array}{l}\text { ¿Cómo evalúas tu formación pre-terciaria? } \\
\text { ¿Consideras que tu preparación en EMS fue adecuada? }\end{array}$ \\
\hline & & & & \multirow{4}{*}{$\begin{array}{c}\text { Preferencias } \\
\text { y } \\
\text { expectativas } \\
\text { pre- } \\
\text { terciarias. } \\
\text { Eventuales } \\
\text { cambios de } \\
\text { expectativas } \\
\cdot \\
\text { Expectativas } \\
\text { de } \\
\text { culminación } \\
\text { de la } \\
\text { «carrera }\end{array}$} & $\begin{array}{l}\text { Al culminar } 6^{\circ} \text { de EMS, ¿tenías claro qué «carrera» } \\
\text { seguir? ¿Cuál era esa «carrera»? ¿En dónde pensabas } \\
\text { cursarla? }\end{array}$ \\
\hline & & & & & $\begin{array}{l}\text { Si la «carrera» que pensabas seguir no es la que estás } \\
\text { cursando ahora, ¿por qué razón no la cursaste? }\end{array}$ \\
\hline & & & & & $\begin{array}{l}\text { La «carrera» en la que te inscribiste al egresar de EMS, } \\
\text { ¿fue la única que cursaste? } \\
\text { [Si responde negativamente:] ¿A qué se debió ese } \\
\text { cambio? }\end{array}$ \\
\hline & & & & & $\begin{array}{l}\text { Al momento de inscribirte en ES, ¿tu expectativa era } \\
\text { culminar la «carrera»? }\end{array}$ \\
\hline & & & & $\begin{array}{c}\text { Razones de } \\
\text { la } \\
\text { persistencia }\end{array}$ & $\begin{array}{l}\text { ¿En algún momento has pensado en abandonar tus } \\
\text { estudios o en cambiar de «carrera»? ¿Por qué? } \\
\text { ¿Qué fue lo que te mantuvo estudiando? }\end{array}$ \\
\hline & & & & & \\
\hline & & & & $\begin{array}{l}\text { Motivos de } \\
\text { la elección } \\
\text { de la } \\
\text { «carrera» }\end{array}$ & $\begin{array}{l}\text { Formulación en modo interrogativo de las } 7 \text { opciones } \\
\text { presentadas en el formulario de NSSE (ver } \\
\text { «Apéndice» 2). }\end{array}$ \\
\hline & & & & $\begin{array}{c}\text { Motivación } \\
\text { hacia la «ca- } \\
\text { rrera» } \\
\text { elegida }\end{array}$ & $\begin{array}{l}\text { Formulación en modo interrogativo de las } 3 \text { opciones } \\
\text { presentadas en el formulario de NSSE (ver } \\
\text { «Apéndice» 2). }\end{array}$ \\
\hline & & & & $\begin{array}{l}\text { Exigencia } \\
\text { académica }\end{array}$ & $\begin{array}{l}\text { Formulación en modo interrogativo de las } 3 \text { opciones } \\
\text { presentadas en el formulario de NSSE (ver } \\
\text { «Apéndice» 2). }\end{array}$ \\
\hline & & & & $\begin{array}{c}\text { Modalidades } \\
\text { de } \\
\text { aprendizaje } \\
\text { activo }\end{array}$ & $\begin{array}{l}\text { Formulación en modo interrogativo de las } 5 \text { opciones } \\
\text { presentadas en el formulario de NSSE (ver } \\
\text { «Apéndice» 2). }\end{array}$ \\
\hline & & & & $\begin{array}{c}\text { Modalidades } \\
\text { de } \\
\text { aprendizaje } \\
\text { colaborativo }\end{array}$ & $\begin{array}{l}\text { Formulación en modo interrogativo de las } 4 \text { opciones } \\
\text { presentadas en el formulario de NSSE (ver } \\
\text { «Apéndice» 2). }\end{array}$ \\
\hline & & & & $\begin{array}{l}\text { Interacción } \\
\text { docentes- } \\
\text { estudiantes }\end{array}$ & $\begin{array}{l}\text { Formulación en modo interrogativo de las } 6 \text { opciones } \\
\text { presentadas en el formulario de NSSE (ver } \\
\text { «Apéndice» 2). }\end{array}$ \\
\hline
\end{tabular}




\begin{tabular}{|l|l|l|l|}
$\mid$ & Trabajo & $\begin{array}{l}\text { ¿Trabajas en forma remunerada? ¿Cuántas } \\
\text { horas/semana? } \\
\text { ¿Ese trabajo está vinculado a la carrera que estás } \\
\text { cursando? }\end{array}$ \\
\cline { 2 - 3 } & $\begin{array}{l}\text { Beca de } \\
\text { apoyo }\end{array}$ & $\begin{array}{l}\text { ¿Tienes alguna beca monetaria o de alojamiento? } \\
\text { iConsideras que eso ha tenido alguna influencia en el } \\
\text { hecho de que hayas continuado estudiando? }\end{array}$ \\
\hline
\end{tabular}




\section{APÉNDICE 2}

(B) Tabla de validación técnica de la pauta de la entrevista en profundidad aplicada a los informantes del «tipo» (v)

\begin{tabular}{|c|c|c|c|c|c|}
\hline \multicolumn{2}{|c|}{$\begin{array}{l}\text { OBJETIVO DE } \\
\text { INVESTIGA- } \\
\text { CIÓN }\end{array}$} & $\begin{array}{l}\text { TÉCNICA } \\
\text { APLICAD } \\
\text { A }\end{array}$ & $\begin{array}{l}\text { INSTRU- } \\
\text { MENTO } \\
\text { APLICADO }\end{array}$ & $\begin{array}{l}\text { VARIABLES } \\
\text { CONSIDE- } \\
\text { RADAS }\end{array}$ & INDICADORES (o PREGUNTAS) \\
\hline \multirow[t]{16}{*}{ (b) } & \multirow{16}{*}{$\begin{array}{c}\text { Identificar } \\
\text { y analizar } \\
\text { las } \\
\text { principales } \\
\text { característi } \\
\text {-cas de las } \\
\text { condiciones } \\
\text {, } \\
\text { situaciones } \\
\text { y } \\
\text { circunstan- } \\
\text { cias que } \\
\text { más inciden } \\
\text {-y el modo } \\
\text { diferencial } \\
\text { en que lo } \\
\text { hacen- en } \\
\text { la decisión } \\
\text { de } \\
\text { abandono } \\
\text { voluntario } \\
\text { de los } \\
\text { estudios en } \\
\text { el 1 }{ }^{\text {er }} \text { año de } \\
\text { la ES en } \\
\text { cada uno de } \\
\text { los tres } \\
\text { centros } \\
\text { educativos } \\
\text { considerad } \\
\text { os }\end{array}$} & \multirow{16}{*}{$\begin{array}{c}\text { Entrevista } \\
\text { en } \\
\text { profundidad } \\
\text { a muestra de } \\
24 \\
\text { informantes } \\
\text { del tipo (v): } \\
8 \text { jóvenes de } \\
\text { cada uno de } \\
\text { los } 3 \text { centros } \\
\text { considerado } \\
\text { s que } \\
\text { iniciaron allí } \\
\text { sus estu- } \\
\text { dios en } 2014 \\
\text { y abandona- } \\
\text { ron sus estu- } \\
\text { dios antes } \\
\text { de } \\
\text { transcurrido } \\
\text { s } 3 \text { meses } \\
\text { del inicio } \\
\text { del } 3 \text { er año } \\
\text { lectivo. } \\
\text { [La } \\
\text { aplicación de } \\
\text { estas entre- } \\
\text { vistas es pos- } \\
\text { terior a la en- } \\
\text { cuesta censal } \\
\text { a estos } \\
\text { informan-tes, } \\
\text { y en la que se } \\
\text { aplicó el } \\
\text { módulo del } \\
\text { formulario } \\
\text { de la } \\
\text { National } \\
\text { Survey of } \\
\text { Student } \\
\text { Engagemen } \\
\text { t] }\end{array}$} & \multirow{11}{*}{$\begin{array}{c}\text { Pauta de } \\
\text { semi- } \\
\text { estructuració } \\
\text { n de } \\
\text { entrevista en } \\
\text { profundidad } \\
\\
\end{array}$} & \multirow{2}{*}{$\begin{array}{l}\text { Trayectoria } \\
\text { y } \\
\text { preparación } \\
\text { pre-terciaria }\end{array}$} & $\begin{array}{l}\text { ¿Dónde cursaste } 6^{\circ} \text { año de EMS? ¿En qué orientación? } \\
\text { ¿Recuerdas por qué elegiste esa orientación? }\end{array}$ \\
\hline & & & & & $\begin{array}{l}\text { ¿Cómo evalúas tu formación pre-terciaria? } \\
\text { ¿Consideras que tu preparación en EMS fue adecuada? }\end{array}$ \\
\hline & & & & \multirow{4}{*}{$\begin{array}{c}\text { Preferencias } \\
\text { y } \\
\text { expectativas } \\
\text { pre- } \\
\text { terciarias. } \\
\text { Eventuales } \\
\text { cambios de } \\
\text { expectativas } \\
\cdot \\
\text { Expectativas } \\
\text { de culmina- } \\
\text { ción de la } \\
\text { «carrera» }\end{array}$} & $\begin{array}{l}\text { Al culminar } 6^{\circ} \text { de EMS, ¿tenías claro qué «carrera» } \\
\text { seguir? ¿Cuál era esa «carrera»? ¿En dónde pensabas } \\
\text { cursarla? }\end{array}$ \\
\hline & & & & & $\begin{array}{l}\text { Si la «carrera» que pensabas seguir no es la que estás } \\
\text { cursando ahora, ¿por qué razón no la cursaste? }\end{array}$ \\
\hline & & & & & $\begin{array}{l}\text { La «carrera» en la que te inscribiste al egresar de EMS, } \\
\text { ¿fue la única que cursaste? } \\
\text { [Si responde negativamente:] ¿A qué se debió ese } \\
\text { cambio? }\end{array}$ \\
\hline & & & & & $\begin{array}{l}\text { Al momento de inscribirte en ES, ¿tu expectativa era } \\
\text { culminar la «carrera»? }\end{array}$ \\
\hline & & & & \multirow{2}{*}{$\begin{array}{l}\text { Motivos de } \\
\text { la elección } \\
\quad \text { de la } \\
\text { «carrera» }\end{array}$} & \\
\hline & & & & & $\begin{array}{l}\text { Formulación en modo interrogativo de las } 7 \text { opciones } \\
\text { presentadas en el formulario de NSSE (ver } \\
\text { «Apéndice» 2). }\end{array}$ \\
\hline & & & & $\begin{array}{l}\text { Motivación } \\
\text { hacia la «ca- } \\
\text { rrera» } \\
\text { elegida }\end{array}$ & $\begin{array}{l}\text { Formulación en modo interrogativo de las } 3 \text { opciones } \\
\text { presentadas en el formulario de NSSE (ver } \\
\text { «Apéndice» 2). }\end{array}$ \\
\hline & & & & $\begin{array}{l}\text { Exigencia } \\
\text { académica }\end{array}$ & $\begin{array}{l}\text { Formulación en modo interrogativo de las } 3 \text { opciones } \\
\text { presentadas en el formulario de NSSE (ver } \\
\text { «Apéndice» 2). }\end{array}$ \\
\hline & & & & $\begin{array}{l}\text { Modalidades } \\
\text { de } \\
\text { aprendizaje } \\
\text { activo }\end{array}$ & $\begin{array}{l}\text { Formulación en modo interrogativo de las } 5 \text { opciones } \\
\text { presentadas en el formulario de NSSE (ver } \\
\text { «Apéndice» 2). }\end{array}$ \\
\hline & & & & $\begin{array}{l}\text { Modalidades } \\
\text { de } \\
\text { aprendizaje } \\
\text { colaborativo }\end{array}$ & $\begin{array}{l}\text { Formulación en modo interrogativo de las } 4 \text { opciones } \\
\text { presentadas en el formulario de NSSE (ver } \\
\text { «Apéndice» 2). }\end{array}$ \\
\hline & & & & $\begin{array}{l}\text { Interacción } \\
\text { docentes- } \\
\text { estudiantes }\end{array}$ & $\begin{array}{l}\text { Formulación en modo interrogativo de las } 6 \text { opciones } \\
\text { presentadas en el formulario de NSSE (ver } \\
\text { «Apéndice» 2). }\end{array}$ \\
\hline & & & & \multirow{3}{*}{$\begin{array}{l}\text { Sobre el } \\
\text { abandono de } \\
\text { los estudios: } \\
\text { razones, }\end{array}$} & $\begin{array}{l}\text { ¿Cuál fue la principal razón que te llevó a interrumpir } \\
\text { tus estudios? }\end{array}$ \\
\hline & & & & & ¿Tu abandono es transitorio o definitivo? \\
\hline & & & & & ¿Tu abandono fue voluntario o no voluntario? \\
\hline
\end{tabular}




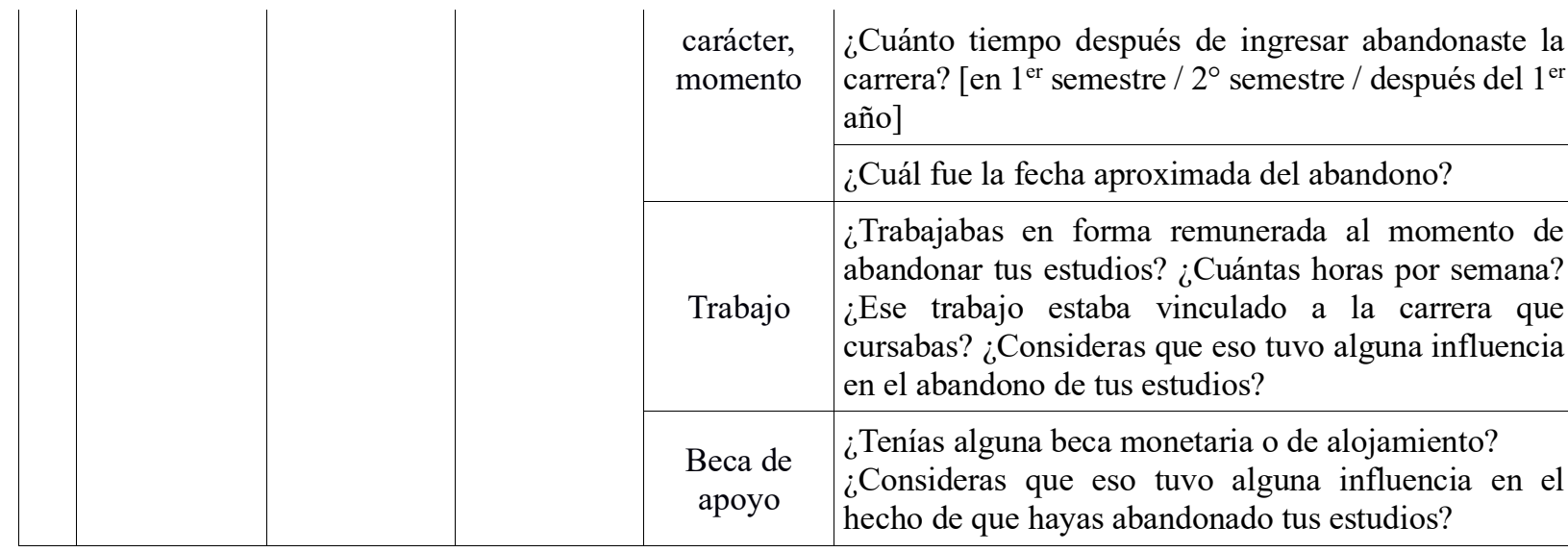

